\title{
CONTINUOUS INTEGRATION OF FAAS DRIVEN BY QUALITY ATTRIBUTES
}

\author{
Pablo Serrano-Gutierrez \\ University of Málaga, Spain
}

\begin{abstract}
The expansion of the cloud has led to the development of serverless applications in which there is no need to be concerned about infrastructure, that can be considered as a service provider in the form of functions, which is known as FaaS (Function as a Service). The development of these applications consists of implementing independent functions that will communicate with each other. This work aims to explore how to combine FaaS and the continuous integration philosophy of DevOps so that applications operators can continuously re-deploy different implementations of functions in order to meet some quality of service requirements, such as latency or energy consumption.
\end{abstract}

\section{KEYWORDS}

Serverless, FaaS, Function as a Service, DevOps, Continuous Integration, Quality of Service

\section{MOTIVATION}

Serverless architecture (Allen, S. et al, 2018), provides the ability to develop and run applications without the need to be concerned about managing the necessary infrastructure for it. This type of architecture represents an evolution of architectures based on microservices (Thönes, J., 2015), in which an application is built on to a set of small services that are deployed and run seamlessly independent, and implement full functionality. Serverless maintains this decomposition approach, but freeing the team from the task of preparation, configuration and maintenance of the servers that form part of the infrastructure in which each and every one of the defined functions are deployed. In this way, when creating an application, it is possible to focus solely on the development and deployment of each of these functions.

To develop a serverless application, as with any other type of application, from the point of view of software engineering, it is necessary to use a certain development methodology. The DevOps (Ebert,C. et al, 2016) philosophy, which has been implemented in the work teams of large technology companies in recent years, makes use of automation, continuous integration and monitoring tools. On the one hand, all the necessary steps to configure the components of the infrastructure are easily reproduced by the automation of the process. The use of continuous integration and continuous delivery tools makes possible to speed up the process of recompilation, testing and deployment of the products in the infrastructure while they are being modified. Finally, based on the data obtained through monitoring, it is possible to make the appropriate decisions on the development and integration process that is taking place, adapting the processes of both developers and operators, to achieve stable products in the shortest time possible. Currently, there are many companies that have decided to migrate their applications to an operating scheme based on the use of serverless functions motivated by the advantages offered by the cloud architecture. Similarly, DevOps has been established as the most common practice in work teams (Balalaie, A. et al, 2016). However, the use of generic tools does not meet the specific needs of serverless applications (Ivanov, V. and Smolander, K., 2018; Debroy, V. and Miller, S., 2020). Therefore, to apply DevOps to the development of serverless applications, more specific tools and methods are needed.

The adoption of a serverless architecture poses some challenges, since an application not only must provide a certain functionality, but it must meet other non-functional requirements, such as latency, availability or security that depends on how well the current deployed functions are behaving. One of the non-functional requirements that is more challenging in the engineering of these systems is latency, especially in applications where it can be a decisive factor, like real-time and IoT applications. 
Challenges in the engineering of serverless applications are not only limited to the achievement of non-functional requirements. Other important challenge in the engineering of these applications is the testing. The ephemeral nature of functions and features of the cloud infrastructure such as the auto-scaling, make impossible the conduction of system-wide tests. Finally, the optimal configuration of the cloud infrastructure is extremely challenging due to the huge quantity of services that are involved.

\section{OBJECTIVES}

The DevOps philosophy is supported by a great variety of tools that facilitate the continuous development and integration of systems. These tools mainly serve two purposes, to automate the deployment and the collection of information about the running system, and the development/integration process. The objective is to use the collected information to improve the different processes involved in development and operation. This permits DevOps teams to decide on the suitability of one or another solution based on a desired quality of service. In the context of serverless applications, these decisions can be the most convenient number of replicas, or the identification of bottlenecks in the execution of the application. Despite there are many frameworks to work with serverless functions (Mohanty, S.K. et al., 2018) and monitoring tools, there is a lack of systems that support rapid decision making as it is required in DevOps environments. This kind of tools will determine, for example, the number of replicas of a specific function or when a specific function should be optimized or replaced by another implementation to guarantee a certain quality of service.

As we stated, the functionality of an application that follows the serverless architectural style is divided into different independent units called serverless functions. So, the functionality of the application is the result of the interaction between these independent functions. The decomposition of the serverless application functionality will have a huge impact on the non-functional requirements of the application (Fritzsch, J. et al., 2019), like execution time and latency. On the other hand, a functional decomposition does not have to be somewhat rigid, but it is possible to use adaptation rules that allow to alternate between different functional decompositions according to the execution context. Our objective is to study how, acting on serverless frameworks, it is possible to optimize the operation of an application according to its specific needs, and also, how an adequate functional decomposition can contribute to the fulfillment of the non-functional requirements of the system, under different execution conditions. Nevertheless, it is necessary to bear in mind that our objective is not to carry out the functional decomposition of a monolithic system to obtain a serverless system, since it is a widely discussed topic that is beyond the purposes of our research. However, it is interesting to consider how this decomposition can affect the application's quality of service requirements.

\section{RESEARCH QUESTIONS}

In order to achieve these objectives, it is necessary to answer several research questions:

RQ1: Is it possible to develop a system that ensures that a serverless application will meet a certain quality of service? An important consideration when creating an application is to ensure that it meets certain non-functional requirements, such as response times, resource consumption, etc. The same consideration deserves a serverless application. Therefore, it will be studied what are the mechanisms provided by serverless frameworks to address this problem and how to use them. In this way, it will be possible to find what frameworks are valid for our purposes and what are more suitable. This is essential, since we are focusing on serverless development instead of microservices in general, so our only connection with the hardware is made through these frameworks and the achievable results may be limited by them. Their degree of integration with the different tools used for DevOps procedures will also be evaluated.

RQ2: What is the optimal functional decomposition considering the conditions of use? In the case of a serverless application, how it works will depend on how the initial problem is divided into different subproblems that can be solved by executing independent functions. Hence, there may exist many possible functional decompositions that help us to solve the problem, but not all of them have to be equally adequate for each specific case. Indeed, sometimes only part of the functionality is recommendable to implement as a 
serverless function, and these decisions will strongly impact the quality of service of the application. Although the objective of our work is not to carry out the functional decomposition of the system, it is interesting to evaluate its behavior in terms of quality of service.

RQ3: Is it possible to design a mechanism that, automatically or semi-automatically, is able at run time, to determine the adaptation rules that are applicable to the system, based on functional decomposition? Taking into account that, in most cases, it will be possible to find several functional decompositions that solve the same problem, it would be interesting to switch between some of these implementations in real time, so that the system can be adapted to different execution conditions, like the number of users, requests, the current traffic or application load balance. These are additional measures to those taken by the framework, such as scaling. This would allow, for example, to send lower quality content or to skip non-essential steps of a certain procedure, if necessary.

\section{METHODOLOGY}

In order to answer the presented research questions, the development of case studies and the use of testing methodologies will have an important role. Therefore, an applied and empirical research methodology will be used. The intention is to exploit the features of FaaS platforms focusing on freely distributed ones, like OpenFaaS and OpenWhisk, two of the most popular serverless platforms. They provide mechanisms to create and encapsulate serverless functions, as well as to define their behavior in terms of replicas, scaling, etc. Other elements will be necessary to operate this platform, like containers and deployment/orchestration tools. With regard to containers, a container runtime environment will be used (i.e., Docker, the most widely used solution). These containers are managed by orchestration tools that perform actions such as the deployment of containers and the orchestration of the different nodes that constitute the application. We also plan to use free distribution platforms like Kubernetes, the most popular one.

The monitoring of the serverless applications is extremely important to answer the research questions. Therefore, it is necessary to manage monitoring applications, which will collect all the data offered by the serverless platforms (i.e., OpenWhisk or OpenFaas) and will serve to study them graphically later. A service mesh, (i.e., Istio, an extension of Kubernetes) is an application that can perform this role. It implements a dedicated infrastructure layer that allows to control the exchange of data between services. It will be used in the development of the case studies to analyze the different adaptation policies that determine the behavior of the functions.

As can be seen in Figure 1, in serverless development, applications must be designed as a composition of multiple stateless functions that can interact with each other. Such functions can be programmed independently using different languages and can be developed by different teams. The deployment of these functions in the infrastructure in which they will be executed is carried out automatically by the serverless framework. These functions are deployed in containers, thus guaranteeing that they will be able to work in the same way regardless of the physical infrastructure on which they are deployed.

Serverless frameworks are supported by an orchestrator, like Kubernetes, which controls exactly where to place each of the containers on the elements that make up the physical infrastructure. It is also responsible for replicating and maintaining the replicas of the containers, following the guidelines set by the serverless framework, so it is possible to adjust their behavior based on certain parameters of quality of service. Above all this, service mesh platforms like Istio, allow to track and monitor the services maintained by the orchestrator. All this information, which we can obtain from the execution of the application, is extremely useful for the DevOps team in charge. If one of the functions does not reach the desired quality of service, this situation will be detected and the DevOps team can reimplement the function. Figure 1 illustrates this scenario, the current QoS of the application is below the target QoS and the problematic function is f2. So, this function is re-implemented as f2' and integrated in the final application. This feedback loop will be part of the continuous integration process, since it allows the development team to obtain information about the result of their work in an automated way. 


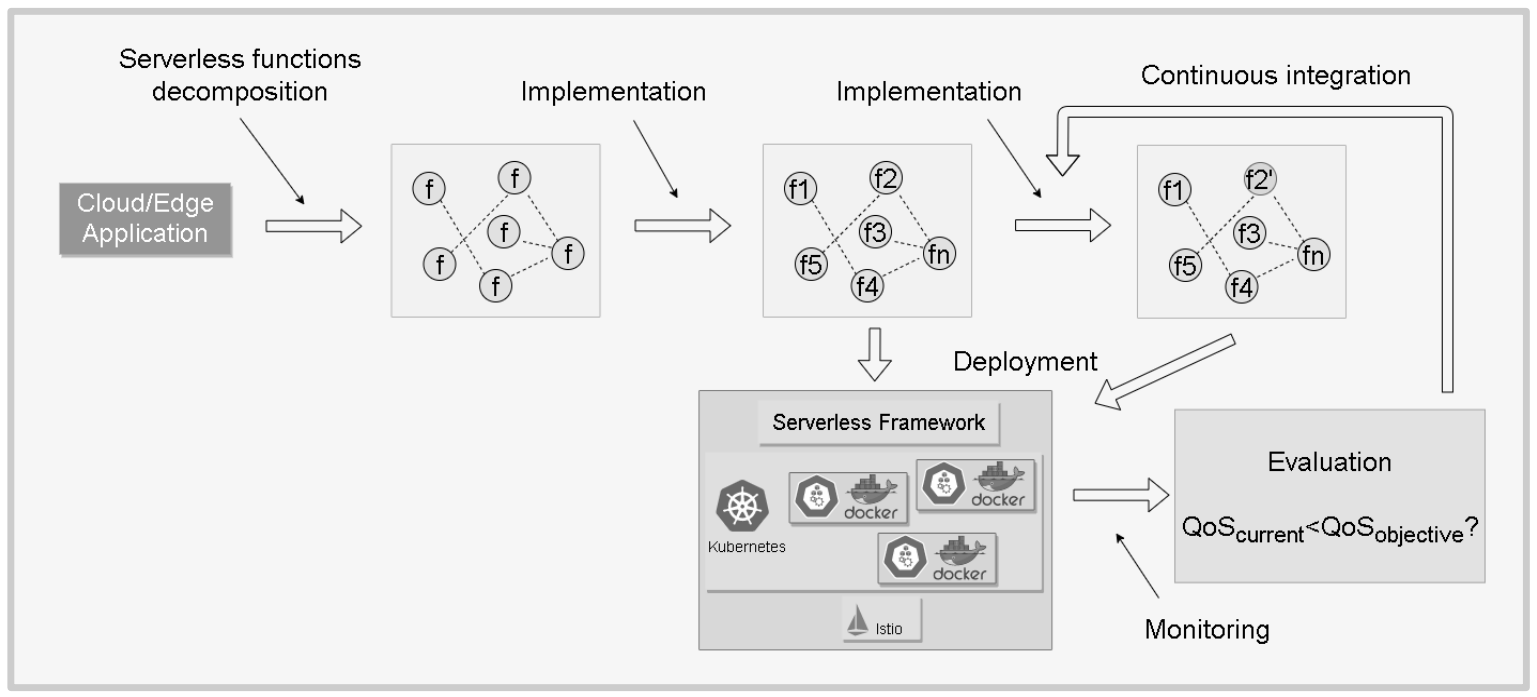

Figure 1. QoS application in serverless development

\section{CURRENT AND FUTURE WORK}

Our recent work is a Systematic Literature Review to know the current degree of integration between serverless development and DevOps practices, as well as existing tools and research works. The study concludes that there is the lack of development in this field and the number of research and publications is low considering the growing interest of the industry. Currently, our research work is focused on implementation tasks, specifically in the inter-operation and integration of the serverless and DevOps tools. The goal is to develop a system able to change the configuration and the deployed serverless function according to the needs of the application at a given time. As part of this work, case studies are being developed to validate this system. The expected results are strategies that can be used by DevOps teams to facilitate the development and operation of serverless-based solutions under certain QoS requirements. Finally, we are working on guidelines to facilitate the decision-making process in order to achieve the optimal decomposition of an application or service into serverless functions taking into account certain requirements.

\section{REFERENCES}

Allen, S. et al, 2018, CNCF WG-Serverless Whitepaper v1.0, Cloud Native Computing Foundation, San Francisco, USA

Balalaie, A. et al, 2016, Microservices Architecture Enables DevOps: An Experience Report on Migration to a Cloud-Native Architecture, IEEE Software,33(3), pp. 2-12.

Debroy, V. and Miller, S., 2020, Overcoming Challenges with Continuous Integration and Deployment Pipelines, IEEE Software,37(3), pp. 21-29.

Ebert,C. et al, 2016, Devops, IEEE Software, 33(3), pp. 94-100.

Fritzsch, J. et al., 2019, From Monolith to Microservices: A Classification of Refactoring Approaches, DEVOPS 2018: Software Engineering Aspects of Continuous Development and New Paradigms of Software Production and Deployment, pp. 128-141.

Ivanov, V. and Smolander, K., 2018, Implementation of a DevOps Pipeline for Serverless Applications, 19th International Conference PROFES 2018, Wolfsburg, Germany, pp. 48-64.

Mohanty, S.K. et al., 2018, An Evaluation of Open Source Serverless Computing Frameworks, IEEE International Conference on Cloud Computing Technology and Science, Nicosia, Cyprus

Thönes, J., 2015, Microservices, IEEE Software, 32(1), pp. 113-116. 